IT in Industry, Vol. 9, No.2, 2021

PublishedOnline12April 2021

\title{
REFLECTO - A SMART MIRROR STRUCTURE WITH A CONTEXTUAL ASSOCIATION OF INTERNET OF THINGS
}

\author{
Md Shahbuddin', Arib Nawaz Khan², Kukatlapalli Pradeep Kumar ${ }^{3 *}$, Cherukuri Ravindranath \\ Chowdary ${ }^{4}$, Michael Moses T ${ }^{5}$, Vinay Jha Pillai ${ }^{6}$ \\ 12345 Department of Computer Science and Engineering, School of Engineering and Technology, \\ ${ }^{6}$ Department of Electronics and Communication Engineering, School of Engineering and Technology \\ CHRIST (Deemed to be University), \\ Bengaluru, Karnataka, India \\ e-mail: md.shahbuddin@btech.christuniversity.in, arib.nawaz@btech.christuniversity.in, kukatlapalli.kumar@christuniversity.in, \\ cherukuri.ravindranath@christuniversity.in,michael.moses@christuniversity.in, vinay.pillai@christuniversity.in \\ *Corresponding Author: kukatlapalli.kumar@christuniversity.in
}

\begin{abstract}
Two of the most valued qualities in the modern-day society are punctuality and presentation. The mission helps to develop the above-mentioned personalities. We have developed an artificial intelligence-based smart mirror that helps in reminding the user about their day to day schedule while they spend their time in front of the mirror. This way the user is ensured of comfort in accessing the basic services with a minimum hinderance. The basic function that our prototype is assumed to carry out is to display user their personalised information like date, time, weather forecast, upcoming holidays or saved events and news
\end{abstract}

\section{INTRODUCTION}

In the hectic life of human race, it can get difficult to be up-to-date about current affairs and to maintain a timely schedule while preparing for the day. Every day in the morning, we end up somehow in front of a mirror while getting ready, additionally, information such as weather conditions and personal schedule can determine the day's preparation. It is a challenge to find all this information in one place without affecting the tasks that are performed in front of the mirror. The main goal is to design a product which can help in easily accessing this information and carry out their routine at the same time. This product should help in enhancing productivity of a person as well as provide a functional and enjoyable experience. One of the objectives of this work is to also provide an optimized and cost effective product to the customers and users of smart mirror. Considering all the parameters and literature work available, a mirror of a unique kind is implemented with all relevant hardware and software components. Rigorous testing is also performed on the executed modules of the research work. bulletin. Face recognition feature is also added in order to identify the user using the camera placed behind the mirror. Because of the face recognition feature the mirror will be able to display personalised data that the identified user wants to know. Reflecto can be easily built by using a two-way mirror, concealed LED screen, microphone and camera to make it interactive. The mirror's functionalities have been extended by integrating it with Google assistant to access IoT devices that have been enabled with home automation.

Keywords - Smart mirror, IoT, Raspberry pi, Artificial intelligence, Anaconda, Python, Google assistant

\section{RELATED WORK}

Design of Smart Mirror Based on Raspberry Pi

In current work, a smart mirror associated on raspberry pi technology is designed and developed for a domestic home environment with the usage of Internet of things. The user can network with the mobile phone over the APP screen, the SYN6288 screen via speech used to make sounds, such as giving command to the screen for time, news, weather on to the screen and find a compatible data network and communicate. This intended mirror has the benefits of bring small in size, easy to operate, inexpensive, and is ideal for families, and has wide solicitation prospects.

\section{Implemented Using Raspberry Pi}

Smart mirror is the rising conception during the fast-changing information technology world. Smart mirror devices have the good potential and future scope for planning the advanced sensible home. 
Table I: Literature analysis featuring smart mirror contexts based on domain and research gap

\begin{tabular}{|c|c|c|c|c|c|}
\hline Name & Author & Year & Domain & Smart Mirror Aspect & Research gap/ Notes \\
\hline [1] Design of smart mirror & Sun Yong & 2018 & Automation & Yes & No Face recognition \\
\hline [2] voice controlled smart mirror & Adokiye Charles & 2018 & Security & Yes, Voice controlled & Does not have AI \\
\hline $\begin{array}{l}\text { [3] Smart mirror E health } \\
\text { Assistance }\end{array}$ & Biljana Cvetkosa & 2017 & Health & Yes & Not feasible \\
\hline $\begin{array}{l}\text { [4] A study on user identification } \\
\text { and }\end{array}$ & Mun H. J & 2019 & Security Privacy & Yes, Face recognition & Not voice controlled \\
\hline $\begin{array}{l}\text { [5 ]SmiWork-Interactive smart } \\
\text { mirror }\end{array}$ & Oihane Gomez & 2018 & Health & $\begin{array}{l}\text { Yes, RFID } \\
\text { authentication }\end{array}$ & User's privacy \\
\hline $\begin{array}{l}\text { [6] Smart mirror -Digital } \\
\text { magazine for university }\end{array}$ & R. Akshaya, S.Gowri & 2018 & IoT & Yes & $\begin{array}{l}\text { Not voice controlled, no } \\
\text { AI }\end{array}$ \\
\hline $\begin{array}{l}\text { [7] Home Automation -smart } \\
\text { mirror }\end{array}$ & Sakthivel A & 2019 & Automation & Yes, & $\begin{array}{l}\text { Not voice controlled, no } \\
\text { AI }\end{array}$ \\
\hline $\begin{array}{l}\text { [8] Smart mirror -An AI personal } \\
\text { assist }\end{array}$ & Sridhar SK & 2019 & $\begin{array}{l}\text { Artificial } \\
\text { Intelligence }\end{array}$ & Yes, Face recognition & $\begin{array}{l}\text { User's privacy, not } \\
\text { feasible }\end{array}$ \\
\hline $\begin{array}{l}\text { [9] Smart mirror- Interactive } \\
\text { display }\end{array}$ & $\begin{array}{l}\text { Athira S, Frangly } \\
\text { francis }\end{array}$ & 2016 & IoT & Yes & $\begin{array}{l}\text { Not voice controlled, no } \\
\text { AI }\end{array}$ \\
\hline $\begin{array}{l}\text { [10] Recognizing Human Face } \\
\text { and Voice }\end{array}$ & $\begin{array}{l}\text { Koushiki } \\
\text { Mukhopadhyay }\end{array}$ & 2018 & $\begin{array}{l}\text { Artificial } \\
\text { Intelligence }\end{array}$ & $\begin{array}{l}\text { Yes, Voice \& Face } \\
\text { recognition }\end{array}$ & User's privacy \\
\hline
\end{tabular}

Mirror may be simply interacted by the residents and is ready to manage the house appliances and conjointly experience the AI chat assistant. Smart mirrors area unit a replacement addition to IoT artefact group that has been gaining an outstanding arrangement of courtesy in current years by every manufacturing sector. Mirror consists of functionalities like real time info and information updates, voice commands, face recognition.

To manifest a user victimization the voice recognition module, the mike is turned on and also the user is prompted to mention the pass phrase saved throughout registration. Automatic face recognition technology adopts a picture matching approach to spot people. This model promotes eudemonia and healthier lifestyles within the work environment through some algorithms.

Additionally, psychological feature recommendation associated with physical performance is provided via invitation by relating speech-based acknowledgment methods. End-users may query the glass regarding their ranking location during a fair-daily competition that offers social gratitude to the most-active-user. Table I depicts the literature study made on existing smart mirror mechanism with various scholars working on the same. Smart mirror aspect and research gap found is also addressed in the table along domain. The first column consists of mirror aspect name with respect to authors vision.

\section{MeThODOLOGY}

Artificial Intelligence was a theoretical understanding and is a technology in today's era. It is not only seen in robotics but also in every industry. It plays a key role in the industry revolution 4.0 as far as automation is concerned. This intelligence is based on heuristic approach followed with lot of historical data available in hand. Prediction is possible by analysing the data in an appropriate way. AI concepts are used in the work carried out with respect to capturing of realtime time and providing needed information to the user of the system.

Convolutional Neural Network (CNN) is Deep Learning formula which may absorb an input image, assign importance to varied aspects/objects within the image and be ready to differentiate one from the other. They have applications in image and video recognition, recommender sys-teems, image classification, medical image analysis, natural language pro-cessing, and financial time series Let's take face detection as associate degree example. Initially, the formula desires heap of positive pictures of faces and negative pictures while not faces to coach the classifier. Then we'd like to extract options from it.

First step is to gather the Haar options. A Haar feature considers adjacent rectangular regions at a selected location in a very detection window, sums up the constituent intensities in every region and calculates the distinction between these sums. Fig 1 shows the three aspects of features which are as follows.
a) Edge Feature
b) Line Feature and 


\section{c) Four Rectangle Feature}

The local Binary Pattern histogram (LBPH) formula may be straight forward answer on face recognition problem, which may acknowledge each front face and side face.

Fig 2 shows the use case understanding of the smart mirror scenario. It has the following features mentioned as use cases. Mirror data base, weather report information, trouble shoot use case by admin are some of the important use cases. The actors are connected with use cases via association relationship.
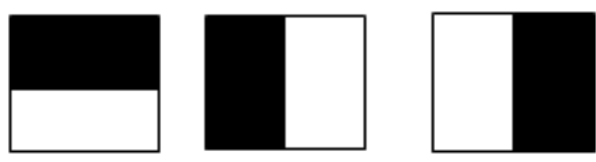

(a)

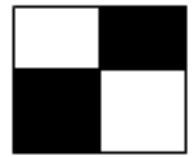

(c)

Fig 1: (a) Edge Feature (b) Line Feature and (c) Four Rectangle Feature

The set of use cases considered in this regard are as follows.
a. Smart Mirror DB
b. Weather Report scenario
c. Day-time display
d. Scheduler
e. Troubleshooting

Actors playing on the system provided here are as follows:

a. Mirror daily users

b. Admin

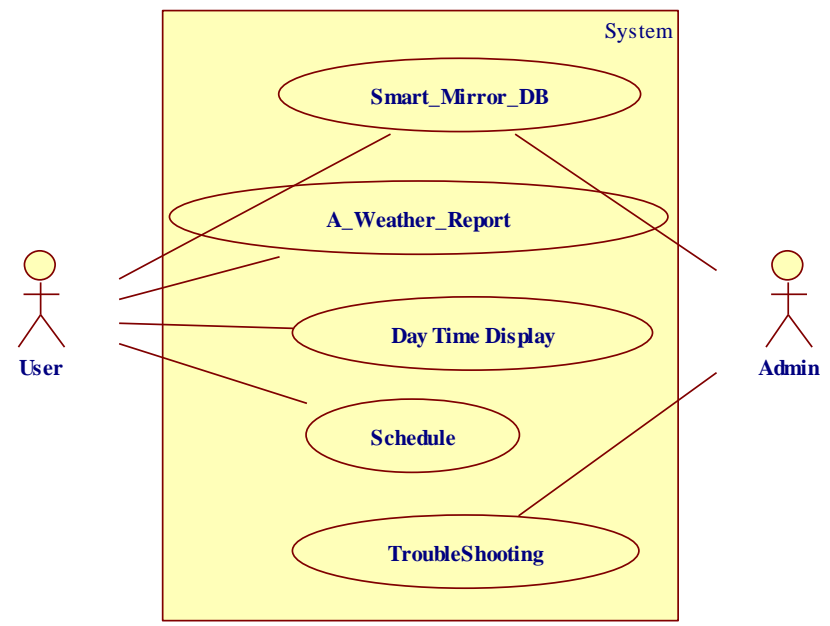

Fig 2: Use case scenarios of smart mirror model
When the schemes were combined, it absolutely was attainable to point out that the system met the planned needs that were set at the start of the development. the web and boundary subsystems are ready to be swapped between, that means that the handler will simply switch the settings after they need and have the system connect with the web and break down the information to show once the mirror is in traditional operation.

The On/Off scheme testing incontestable that the ability to the show was ready to be organised by program of the user, permitting the mirror to show itself on once someone is standing ahead of it. The emotion detection was tested and the range of emotions found in the output summed up to 100.

\section{Hardware requirements}

Two-way mirror: This is most likely the foremost necessary portion of the hardware as a result of its accountable for making the futurist impact and is the biggest part of the smart mirror.

Raspberry $\mathrm{Pi}$ 3: The Raspberry $\mathrm{Pi}$ is a single-board pc technologically advanced by the Raspberry $\mathrm{Pi}$ foundation within the United Kingdom.

Display: For the display a 15-inch monitor with built in speakers and a HDMI cable is employed to attach the show to the Raspberry Pi for audio and video.

Microphones: A microphone is attached to influence the voice acknowledgment competences of the device.

Frame and support: The structure is created of wood and it delivers the provision for the glass. It frames the glass and offers the way for hanging the glass on a wall. It is like a wooden box in which we can place the monitor, raspberry pi and other components.

\section{Software requirements}

Python: It is an construed, advanced, all-purpose programming language. Python is dynamically written. This programming language provisions manifold programming patterns as well as practical, object adapted to various scenarios.

Anaconda: It is AN ASCII text file IDE that helps in simple usage of the Python and $\mathrm{R}$ programming language, it helps in simplifying package management and deployment.

Jupyter Notebook: The Jupyter Notebook is an ASCII text file internet application that enables you to form and share documents that contains live code, equations, visualizations and narrative text.

Raspberry Pi package strategy: Raspberry pi core package is coded to mistreat python, this platform will raise varied Linux operating system.

Computer code supported network. 
TKinter: It is the quality graphical user interface collection for python. Python once united with TKinter delivers a firm and relaxed way to produce graphical user interface solicitations. Tkinter delivers a strong object concerned with the TK graphical user interface package.

When the systems were integrated, it absolutely was attainable to point out that the system met the planned needs that were set at the start of the research work. The internet and interface subsystems are ready to be switched between, that means that the user will switch the setting after they need and have the system connect with the web and break down the info to show once the mirror is in operation.

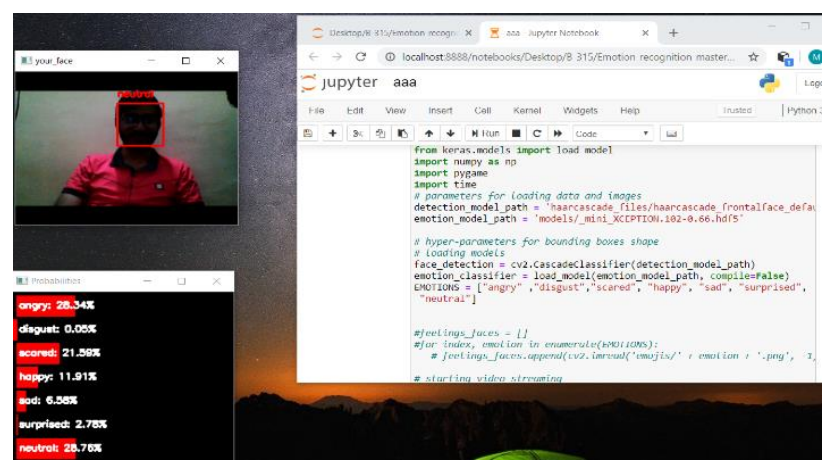

Fig 3: Features and emotion detection mechanism

\section{RESUltS AND DISCUSSION}

The Reflecto - an IoT based smart glass system established syndicates the ideas and procedures that are executed in different structures like mobile smart devices. it's a completely unique methodology of making a smart collaborating arrangement that's consistent and simple to use. These services of the smart Mirror are often more extended to support future enhancements and developments. this implies that options like drawn-out home automation (control close lighting systems, centralized ACs systems, and other standard home computerisation systems/devices like Google Home) and extra ap-plications and widgets are often enforced. User's customized knowledge like calendar, news feeds, and different data pertinent to their mode are enforced and displayed. The smart Glass uses speech instructions to change between every read, and accomplishes the utilities matching to the feedback speech facility. Instead of confining to a home, we will implement the practicality of our smart Glass structure to commercial work, and community environments.

When the smart Glass is in execution, voice commands are often cloaked by contextual that's gift within the setting within which the smart Mirror is placed. Emotion detection may be a unique method that aids the user to involuntarily play songs supported the feelings of the user It acknowledges the facial emotions of the user and plays the songs in step with their emotion. This shows the basic mirror display which we get after turning on the display. It shows date and time on the top left, temperature of the set location on the top right along with the forecast. It shows list holidays/appointment made in your google account. At the bottom the news headlines change after a certain amount of time. Fig 3 provides a pictorial understanding of emotion detection seen in the mirror such as anger, happiness, sorrow etc. It also shows the percentage of the same. Fig 4 is a photograph of the smart mirror which was used for experimentation.

The image demonstrates the elementary consumer interface of the smart mirror that will be used by the end user. The user boundary will be showing the information on the glass and the unfilled area amongst will house the image of the handler.

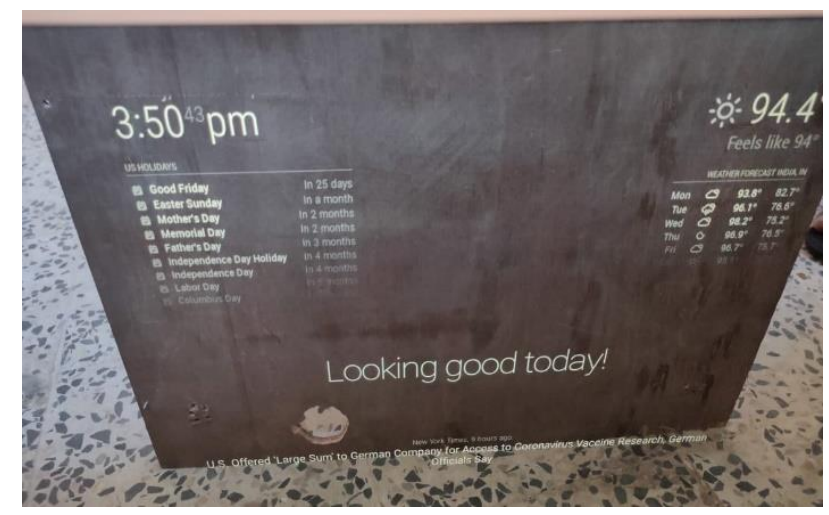

Fig 4: A simple view of smart mirror

This brings out the total cost of our hardware to be INR 7060. The cost estimation was done initially, to provide us an insight of the budget details, and the time required to assemble the components, and arrange for the financial backing. This helped us greatly in the future, as we could bring in the components accordingly, and plan out the course of action. This assisted us to create a timeline before even initiating the work, and helped generate a schedule for the development and fulfilment.

\section{Conclusion AND Future Scope}

Our goal was to provide relevant information to user which can be easily accessed by them while being able to perform their task. We were able to build a working prototype of Reflecto - an IoT based smart mirror which shows the user date and time, weather condition, upcoming events and news bulletin. It is also integrated with Google assistant for voice commands and facial emotion detection. It helps in enhancing user experience by accessing the information from different places and also interacting with user data from the internet. It brings all the relevant information for the user at one place so that it is available to user effortlessly and saves 
their time. By adding touch screen display that has a better display quality it will be more interactive and user will be able to view better graphics.

Smart mirror can become a part of IoT home automation system and become a part of everyday life.

\section{REFERENCES}

[1] Design of Smart Mirror Based on Raspberry Pi: Sun, Y., Geng, L., \& Dan, K. (2018, January). In 2018 International Conference on Intelligent Transportation, Big Data \& Smart City (ICITBS) (pp. 77-80). IEEE.

[2] Yeo, U. C., Park, S. H., Moon, J. W., An, S. W., \& Han, Y. O. (2019). Smart Mirror of Personal Environment using Voice Recognition. The Journal of the Korea institute of electronic communication sciences, 14(1), 199-204.

[3] Mun, H. J. (2019). A Study on the User Identification and Authentication in the Smart Mirror in Private. Journal of Convergence for Information Technology, 9(7), 100-105.

[4] Gomez-Carmona, O., \& Casado-Mansilla, D. (2017, July). SmiWork: An interactive smart mirror platform for workplace health promotion. In 2017 2nd International Multidisciplinary Conference on Computer and Energy Science (SpliTech) (pp. 16). IEEE.

[5] Mun, H. J. (2019). A Study on the User Identification and Authentication in the Smart Mirror in Private. Journal of Convergence for Information Technology, 9(7), 100-105.

[6] Akshaya, R., Raj, N. N., \& Gowri, S. (2018, July). Smart Mirror-Digital Magazine for University Implemented Using Raspberry Pi. In 2018 International Conference on Emerging Trends and Innovations Engineering and Technological Research (ICETIETR)

[7] Sridhar, S. K., Vivek, N. V., AG, B. J., Aishwarya, H. M., \& Akhil, R. SMART MIRROR ARTIFICIALLY INTELLIGENT PERSONAL ASSISTANT.

[8] Mathivanan, M. P., Anbarasan, G., Sakthivel, A., \& Selvam, G. (2019, March). Home Automation Using Smart Mirror. In 2019 IEEE International Conference on System, Computation, Automation and Networking (ICSCAN) (pp. 1-4). IEEE.

[9] Athira, S., Francis, F., Raphel, R., Sachin, N. S., Porinchu, S., \& Francis, S. (2016, March). Smart mirror: A novel framework for interactive display. In 2016 International Conference on Circuit, Power and Computing Technologies (ICCPCT) (pp. 1-6). IEEE.

[10] Mukhopadhyay, K., Sinha, C., Saha, H. N., Rakshit, S., \& Auddy, S. (2018, November). Smart Mirror-a Secured Application of Artificial Intelligence Recognizing Human Face and Voice. In 2018 IEEE 9th Annual Information Technology, Electronics and Mobile Communication Conference (IEMCON) (pp. 1279-1289). IEEE. 